[5] 0. H. Hamilton, A fixed point theorem for pseudo-arcs and certain other metric continua, Proc. Amer. Math. Soc. 2 (1951), pp. 173-174.

[6] - Fixed points under transformations of continua which are not connected in kleinen, Trans. Amer. Math. Soc. 44 (1938), pp. 18-24.

[7] S. Kinoshita, On some contractible continua without fixed point poperty, Fund. Math. 40 (1953), pp. 96-98.

[8] K. Kuratowski, Topologie II, Warszawa 1952

[9] T. van der Walt, Fixed and almost fixed points, Mathematisch Centrum, Amsterdam 1963.

Reçu par la Rédaction le 30. 5. 1967

\section{Post algebras as semantic bases of some many-valued logics}

by

\section{G. Kirin (Zagreb)}

1. Introduction. The subject of the present paper are the manyvalued functional calculi of the first order of Turquette-Rosser (without equality) and the role of Post algebras therein which is fully analogous in many respects to that played by Boolean algebras in the classical twovalued case.

The main results are obtained by means of a certain formalism of Gentzen and the diagram of formulas due to H. Rasiowa and R. Sikorski introduced by them in [6].

The author desires to express his profound indebtendness to Professor H. Rasiowa for the problem itself and for the kindly advice as well.

2. Post algebras. A distributive lattice with zero $\left(e_{0}\right)$ and unit $\left(e_{n-1}\right)$, which contains an $n$-element chain $e_{0}, e_{1}, \ldots, e_{n-2}, e_{n-1}($ with $n \geqslant 2)$ and is such that for each $x$ there are $n y$ 's with the property that

if

$$
x=\left(e_{0} \vee y_{0}\right) \wedge\left(e_{1} \vee y_{1}\right) \wedge \ldots \wedge\left(e_{n-1} \vee y_{n-1}\right)
$$

$$
y_{r} \vee y_{s}=e_{n-1} \quad \text { for } \quad r \neq s \quad \text { and } \quad y_{0} \wedge \ldots \wedge y_{n-1}=e_{0},
$$

is called a Post algebra of order $n\left({ }^{1}\right)$. Together with the additional conditions: for every $x$

and

$$
x \vee e_{i-1}=e_{i} \quad \text { implies } \quad x=e_{i} \quad(i=1, \ldots, n-1)
$$

$$
x \wedge e_{1}=e_{0} \quad \text { implies } \quad x=e_{0},
$$

Post algebras are fully characterized. (Cf. [1] and, for another axiomatization, [7].)

Since the uniqueness of those $y$ 's has been proved in [1], we write henceforth

$$
y_{i}=k_{i}(x) \quad \text { for } \quad i=0, \ldots, n-1 \text {. }
$$

( ${ }^{1}$ One obtains Boolean algebras, for $n=2$, by putting $y_{0}=x$ and $\left.y_{1}=\right\urcorner x$ (the complement of $x$ ). 
The elements $e_{0}, \ldots, e_{n-1}$ are also unique and different, i.e. $e_{i-1}<e_{i}$ for $i=1, \ldots, n-1$, as shown in [1]. In addition to these unary lattice operations we also introduce their duals

$$
z_{i}=j_{i}(x) \quad\left[\text { for } \quad i=0, \ldots, n-1{ }^{(2)} .\right.
$$

Now we rewrite some identities from [1], adjust the proofs of some others or deduce those concerned with the mutual dependence of $j_{i}$ 's and $k_{i}$ 's (as is done in [4]).

$$
\begin{aligned}
& x=k_{0}(x) \wedge\left(e_{1} \vee k_{1}(x)\right) \wedge \ldots \wedge\left(e_{n-2} \vee k_{n-2}(x)\right), \\
& x=\left(e_{1} \wedge j_{1}(x)\right) \vee \ldots \vee\left(e_{n-2} \wedge j_{n-2}(x)\right) \vee j_{n-1}(x) .
\end{aligned}
$$

$$
k_{r}(x) \vee k_{s}(x)=e_{n-1}, \quad j_{r}(x) \wedge j_{s}(x)=e_{0}
$$$$
\text { if } r \neq s \text { and } r, s=0, \ldots, n-1 \text {. }
$$

$$
k_{0}(x) \wedge \ldots \wedge k_{n-1}(x)=e_{0}, \quad j_{0}(x) \vee \ldots \vee j_{n-1}(x)=e_{n-1} .
$$

$k_{r}(x) \vee j_{r}(x)=e_{n-1}, \quad j_{r}(x) \wedge k_{r}(x)=e_{0}, \quad r=0, \ldots, n-1$.

In $(2.5) \dot{r}, s=0, \ldots, n-1$.

$$
k_{r}\left(k_{s}(x)\right)=\left\{\begin{array}{ll}
k_{s}(x) & \text { if } \quad r=0, \\
j_{s}(x) & \text { if } \quad r=n-1, \\
e_{n-1} & \text { otherwise }
\end{array} \quad j_{r}\left(j_{s}(x)\right)=\left\{\begin{array}{lll}
k_{s}(x) & \text { if } r=0, \\
j_{s}(x) & \text { if } r=n-1, \\
e_{0} & \text { otherwise. }
\end{array}\right.\right.
$$

$$
k_{r}\left(j_{s}(x)\right)=\left\{\begin{array}{ll}
j_{s}(x) & \text { if } \quad r=0, \\
k_{s}(x) & \text { if } \quad r=n-1, \\
e_{n-1} & \text { otherwise. }
\end{array} j_{r}\left(k_{s}(x)\right)= \begin{cases}j_{s}(x) & \text { if } \quad r=0, \\
k_{s}(x) & \text { if } r=n-1, \\
e_{0} & \text { otherwise . }\end{cases}\right.
$$

In (2.6) $s=0, \ldots, n-1$.

We cite only that group of identities for the least upper bound which is connected with $k$ operators.

$$
\begin{gathered}
k_{0}\left(\bigwedge_{t \in T} x_{t}\right)=\bigwedge_{t \in T} k_{0}\left(x_{t}\right) \\
k_{r}\left(\bigwedge_{t \in T} x_{t}\right)=\bigwedge_{t \in T} k_{r}\left(x_{t}\right) \vee \bigvee_{i=0}^{r-1} \cdot\left(\bigvee_{t \in T} j_{i}\left(x_{t}\right)\right) \quad \text { for } \quad 1 \leqslant r \leqslant n-2, \\
k_{n-1}\left(\bigwedge_{t \in T} x_{t}\right)=\bigvee_{t \in T} k_{n-1}^{\prime}\left(x_{t}\right) .
\end{gathered}
$$

(2) More precisely, $k_{i}(x)$ and $j_{n-i-1}(x)$ stand in mutual duality. So do $e_{i}$ and $e_{n-i-1}$.
Take herein $T=\{1,2\}$ and write $x_{1}=x, x_{2}=y$. On account of $\bigvee_{i=0}^{r-1} j_{i}(x)$ $=\bigwedge_{i=r}^{n-1} k_{i}(x)$, we can put the identities for $k_{r}(x \wedge y)$ into a more convenient form $\left({ }^{3}\right)$ :

$$
\begin{aligned}
& k_{0}(x \wedge y)=k_{0}(x) \wedge k_{0}(y) \\
& \text { (2.8) } \quad k_{r}(x \wedge y)=\left(k_{r}(x) \vee \bigwedge_{i=r}^{n-1} k_{i}(y)\right) \wedge\left(k_{r}(y) \vee \bigwedge_{i=r}^{n-1} k_{i}(x)\right), \quad 1 \leqslant r \leqslant n-2, \\
& k_{n-1}(x \wedge y)=k_{n-1}(x) \vee k_{n-1}(y) \text {. }
\end{aligned}
$$

3. Syntax. Our intention is to interpret a first order language $A$ in (complete) Post algebras. Its syntax is therefore built up in accordance with lattice operations of Post algebras.

\section{We assume}

$X=\left\{x_{k}\right\}$, for any natural $k$, as the set of free individual variables,

$Y=\left\{y_{k}\right\}$ as the set of bound individual variables,

$C=\left\{c_{k}\right\}$ as the set of individual constants,

$\Phi=\left\{\Phi_{q}^{k}\right\}$, for any natural $q$ and $k$, as the set of functors ( $k$ being the number of free places for terms in some $\Phi_{q}^{k}$ and $q$ the proper index),

$\mathcal{A}=\left\{A_{q}^{k}\right\}$ as the set of elementary propositional functions (with the same meaning of $q$ and $k)$,

$\Sigma=\left\{J_{0}, \ldots, J_{n-1}, K_{0}, \ldots, K_{n-1}^{i}, \cup, \cap\right\}$ as the set of unary and binary propositional functors,

$E=\left\{E_{0}, \ldots, E_{n-1}\right\}$ as the set of propositional constants,

$\Omega=\left\{(,)_{-},-\right\}$as the set of auxiliary sings (the brackets and the down-bounding, resp. up-bounding, quantifier).

The set of terms $\mathcal{C}$ of the language $\Lambda$ is the least set that contains. each $x_{i} \in X$ and each $c_{i} \in C$, and for every $k$-tuple of its elements $t_{i_{1}}, \ldots, t_{i_{k}}$. and each $\Phi_{q}^{k}$ it contains also $\Phi_{q}^{k}\left(t_{i_{1}} \ldots t_{i_{k}}\right)$.

The set of formulas $\mathcal{F}$ of the language $\Lambda$ is the least set that contains. each $E_{i} \in E$ and for every $k$ - tuple of terms $t_{i_{1}}, \ldots, t_{i_{k}}$ it contains $A_{q}^{k}\left(t_{i_{1}}, \ldots, t_{i_{k}}\right)$ for every $A_{q}^{k} \in \mathfrak{t}$. Such formulas are called elementary.

$K_{i}(F)$ and $J_{i}(F)$ are formulas if $F$ is a formula. So are $F \cup G$ and $F \cap G$ if $F$ and $G$ are formulas.

( $\left.{ }^{3}\right)$ For infinite $T$, however, there is no such useful way. Sections 3,4 and 5 of the present paper are strongly influenced by this fact. See particularly the property denoted there by (Syn). 
If there is an occurrence of a certain $x_{j}$ in a formula $F$ and there is no occurrence of $y_{i}$ in it, then $F^{\prime}\left(y_{i} / x_{j}\right)$ denotes the result of the replacement of each occurrence of $x_{j}$ in $F$ by $y_{i}$. Under such conditions $y_{i} F\left(y_{i} / x_{j}\right)$ and $\bar{y}_{i} F\left(y_{i} / x_{j}\right)$ are formulas if $F$ is a formula of the previous kind.

The outermost brackets in a formula will be omitted as usual.

The order of a formula $F$ is the natural number $\gamma\left(F^{\prime}\right)$ defined as follows

$$
\gamma(F)=\left\{\begin{array}{rr}
0 & \text { if } F \text { is of the form } E_{i}, J_{i}\left(A_{q}^{k}\left(t_{i_{1}} \ldots t_{i_{k}}\right)\right) \\
& \text { or } K_{i}\left(A_{q}^{k}\left(t_{i_{1}} \ldots t_{i_{k}}\right)\right), \\
1+\gamma(G) & \text { if } F \text { is of the form } A_{q}^{k}\left(t_{i_{1}} \ldots t_{i_{k}}\right), \\
1+\max \{\gamma(G), \gamma(H)\} & \text { if } F \text { is of the form } K_{i}(G), J_{i}(G), y_{i} G \\
\text { or } \bar{y}_{i} G, & \text { or } G \cap H . H
\end{array}\right.
$$

The proposed operators are functionally complete. Suppose we are given some function $f: I_{n}^{m} \rightarrow I_{n}$, where $m$ is a natural number and $I_{n}=\{0, \ldots, n-1\}$. Every such $f\left(x_{1}, \ldots, x_{m}\right)$ is expressible in terms of the following functions: the constants $0, \ldots, n-1, \max \left(x_{1}, x_{2}\right), \min \left(x_{1}, x_{2}\right)$ and $k_{i}\left(x_{1}\right)(i=0, \ldots, n-1)$ when defined on $I_{n}$ according to (2.5), taking $s$ instead of $e_{s}$, by means of a rule of substitution (of functions or variables for variables) or in terms of the previous ones and $j_{i}(x)$ instead of $k_{i}(x)$. Namely, the perfect normal forms of $f\left(x_{1}, \ldots, x_{m}\right)$ are:

$$
\begin{gathered}
\min _{i_{1}, \ldots i_{m} \in I_{n}}\left(\max \left(k_{i_{1}}\left(x_{1}\right), \ldots, k_{i_{m}}\left(x_{m}\right), f\left(i_{1}, \ldots, i_{m}\right)\right)\right), \\
\underset{i_{1}, \ldots i_{m} \in I_{n}}{\max }\left(\min \left(j_{i_{1}}\left(x_{1}\right), \ldots, j_{i_{m}}\left(x_{m}\right), f\left(i_{1}, \ldots, i_{m}\right)\right)\right) .
\end{gathered}
$$

4. Semantics. Let $Z$ be a non-empty set and $P$ a complete Post algebra of order $n$. Given a certain $v \in Z^{X}(v: X \rightarrow Z$ may be called a nomination), each extension of that $v$ upon $\mathcal{G} \cup \mathcal{F}$ with values in $Z \cup P$ will be called an interpretation $w$ of the language $\Lambda$ if the following conditions are satisfied (cf. [5]):

$$
\begin{aligned}
w\left(x_{i}\right) & =\pi_{i}(v), \quad \text { the } i \text { th coordinate of } v \in Z^{X}, \\
w\left(c_{i}\right) & =z_{w\left(c_{i}\right)}, \\
w\left(\Phi_{q}^{k}\left(t_{i_{1}} \ldots t_{i_{k}}\right)\right) & =\varphi_{q}^{k}\left(w\left(t_{i_{1}}\right), \ldots, w\left(t_{i_{k}}\right)\right),
\end{aligned}
$$

where $\varphi_{q}^{k}$ is thought of as a $k$-ary operation in $Z$ for every $q$ and $k$, i.e. an element of $Z^{\left(Z^{k}\right)}$. Our $w$, restricted to $Z$ and with values in $Z$, is thought
of as the realization of terms of $A$.
For formulas we put:

$w\left(E_{r}\right)=e_{r}^{P}, \quad$ where $e_{r}^{P}$ denotes the $r$ th element of the ascending chain $e_{0}, \ldots, e_{n-1}$ in the algebra $P$,

$w\left(A_{q}^{k}\left(t_{i_{1}} \ldots t_{i_{k}}\right)\right)=a_{q}^{k}\left(w\left(t_{i_{1}}\right), \ldots, w\left(t_{i_{k}}\right)\right), \quad$ where $a_{q}^{k}$, for every $q$ and $k$, is thought of as a $k$-ary many-valued relation in $Z$, i.e. and element of $P^{\left(Z^{k}\right)}$.

$$
\begin{aligned}
& w\left(K_{r}(F)\right)=k_{r}\left(w\left(F^{\prime}\right)\right), \\
& w\left(J_{r}(F)\right)=j_{r}(w(F)), \\
& w(F \cup G)=w\left(F^{\prime}\right) \vee w(G), \\
& w(F \cap G)=w(F) \wedge w(G) .
\end{aligned}
$$

Let $W(w, j)$ denote the set of all interpretations $w^{\prime}$ which coincide with $w$ on $X$ in all coordinates but at most the $j$ th, i.e.

$$
W(w, j)=\left\{w^{\prime}: w^{\prime} /\left(X-\left\{x_{j}\right\}\right)=w /\left(X-\left\{x_{j}\right\}\right)\right\} .
$$

Then let

$$
w\left(\underline{y}_{i} F\left(y_{i}\right)\right)=\inf _{w^{\prime} \in W(w, j)}\left\{w^{\prime}\left(F^{\prime}\left(x_{j} / y_{i}\right)\right)\right\}
$$

for an arbitrary $x_{i} \in X$ which does not occur in the formula $\underline{y}_{i} F\left(y_{i}\right)$,

$$
w\left(\bar{y}_{i} F\left(y_{i}\right)\right)=\sup _{w^{\prime} \in W(w, j)}\left\{w^{\prime}\left(F\left(x_{j} / y_{i}\right)\right)\right\}
$$

under the same conditions on $x_{j}$.

Given $Z, P$ and $w$, the element $w(F) \in P$ is often called the value of the formula $F$ of $\Lambda$ for that $w$. The value of a non-empty sequence of formulas $\mathcal{L}$ is defined as follows

$$
w(\mathcal{L})=\sup \left\{w\left(\boldsymbol{F}_{1}\right), \ldots, w\left(\boldsymbol{F}_{k}\right)\right\}
$$

if $\mathcal{L}$ consists of formulas $F_{1}, \ldots, F_{k}$.

A formula $F$ is said to be $s$-satisfiable $(1 \leqslant s \leqslant n-1)$ if there is a certain $w$ defined upon a certain $Z$ and a certain $P$, of order $n$, such that $w\left(F^{\prime}\right) \geqslant e_{s}^{P}$.

A formula $F$ is said to be $s$-tautologous if $w(F) \geqslant e_{s}^{0}$ for every $w$ defined upon every $Z$ and the Post algebra $P^{0}\left({ }^{4}\right)$.

A formula $F$ is said to be $s$-valid if $w(F) \geqslant e_{s}^{P}$ for every $w$ defined upon every $Z$ and every $P$ of order $n$.

( $\left.{ }^{4}\right)$ Its support consists of a single $n$-element chain $e_{0}^{0}, \ldots, e_{n-1}^{0}$, so that $P^{0}$ plays the role of the two-element Boolean algebra in the two-valued case. 
The diagram of formulas. Our aim is to define for every given formula $F$ of $\Lambda$ exactly one set of sequences of formulas, called the diagram of $F$ and denoted by $\mathcal{D}(F)$, obtained by a systematic decomposition of $F$. Therefore we need a complete list of the rules of decomposition of the following general form:

$$
\frac{\mathfrak{L}_{1}, F, \mathfrak{L}_{2}}{\mathfrak{L}_{1}, G_{1}^{1}, \ldots, G_{r_{1}}^{1}, \mathfrak{L}_{2} ; \ldots ; \mathfrak{L}_{1}, G_{1}^{q}, \ldots, G_{r_{q}}^{q}, \mathfrak{L}_{2}}, \quad q \geqslant 1 .
$$

In (4.8) $\mathfrak{L}_{1}$ is always an indecomposable sequence of formulas (possibly empty), $\mathfrak{L}_{2}$ is an arbitrary sequence, $F$ is the formula to be decomposed and $G_{j}^{i}$ are formulas explicitly given ( $q$ and $G_{j}^{i}$ vary with $F$ and $n$ as well). The sequence $\mathfrak{L}_{1}, G_{1}^{i}, \ldots, G_{r_{i}}^{i}, \mathfrak{L}_{2}$ is called the $i$-th premise of the conclusion $\mathfrak{L}_{1}, F, \mathfrak{C}_{2}$.

Each rule of decomposition has to possess two properties, denoted by (Sem) and (Syn) $\left(^{5}\right)$.

(Sem): The sequence $\mathfrak{L}_{1}, F, \mathfrak{L}_{2}$ is $s$-valid ( $s$-tautologous) if and only if the sequence $\mathfrak{L}_{1}, G_{1}^{i}, \ldots, G_{r_{i}}^{i}, \mathfrak{L}_{2}$ is $s$-valid ( $s$-tautologous) for every $i$.

(Syn): $\gamma(F) \geqslant \gamma\left(G_{j}^{i}\right)$ for every $i$ and $j$.

Therefore we define the formulas of the form $\dot{K_{i}}\left(A_{q}^{k}\left(t_{j_{1}} \ldots t_{j_{k}}\right)\right)$, $J_{i}\left(A_{q}^{k}\left(t_{j_{1}} \ldots t_{j_{k}}\right)\right)$ and $E_{i}$ as indecomposable and others as decomposable. A sequence is said to be indecomposable if it is built up of indecomposable formulas only or if it is empty.

A non-empty sequence of formulas that satisfies at least one of the following 4 conditions is said to be $s$-fundamental if it contains:

(i) two formulas of the form $K_{i}\left(F^{\prime}\right)$ and $K_{j}\left(F^{\prime}\right), i \neq j$,

(ii) two formulas of the form $K_{i}(F)$ and $J_{i}\left(F^{H}\right)$,

(iii) $n$ formulas of the form $J_{0}\left(F^{\prime}\right), \ldots, J_{n-1}\left(F^{\prime}\right)$,

(iv) one formula of the form $E_{i}, i \geqslant s$.

The complete list of all the rules is rather long.

1. The rule of elimination of a universal quantifier (Q). Let the set $\boldsymbol{C}$ of all terms be ordered into a sequence

$$
t_{1}, \ldots, t_{n}, \ldots
$$

and let $x_{j}$ be the first free individual variable in (4.9) which does not occur in any formula of $\mathfrak{L}_{1}, \mathfrak{L}_{2}$ or in the formula $\underline{y}_{i} F^{\prime}\left(y_{i}\right)$. The rule of elimination of the quantifier " - " is the following one:

$$
\frac{\mathfrak{L}_{1}, \underline{y}_{i} F^{\prime}\left(y_{i}\right), \mathfrak{L}_{2}}{\mathfrak{L}_{1}, F^{\prime}\left(x_{j} / y_{i}\right), \mathfrak{L}_{2}}
$$

(s) The property (Sem) is a semantic one and that denoted by (Syn) syntactical.
Since $\gamma\left(\underline{y}_{i} F^{\prime}\left(y_{i}\right)\right)>\gamma\left(F^{\prime}\left(x_{j} / y_{i}\right)\right)$, (Syn) holds true for that rule. The same is true of (Sem) because

$$
w\left(\left(\mathfrak{L}_{1}, \underline{y}_{i} F\left(y_{i}\right), \mathfrak{L}_{2}\right) \leqslant w\left(\mathfrak{\complement}_{1}, F\left(x_{j} / y_{i}\right), \mathfrak{L}_{2}\right)\right.
$$

for every $w$ and $x_{j}$ described above. Namely, $w \in W(w, j)$, whence

$$
w\left(y_{i} F^{\prime}\left(y_{i}\right)\right)=\inf _{w^{\prime} \in W(w, j)}\left\{w^{\prime}\left(F^{\prime}\left(x_{j} / y_{i}\right)\right)\right\} \leqslant w\left(F^{\prime}\left(x_{j} / y_{i}\right)\right) .
$$

On the other hand, if one assumes $w\left(F\left(x_{j}\right)\right) \geqslant e_{s}^{P}$ for any $w$ defined upon a certain $P$ and a certain $Z$, one obtains $w^{\prime}\left(F^{\prime}\left(x_{j}\right)\right) \geqslant e_{s}^{P}$ for each $w^{\prime} \in W(w, j)$ and each given $w$ defined upon that $P$ and that $Z$. Since $P$ is complete, we have

$$
\inf _{w^{\prime} \in W(w, j)}\left\{w^{\prime}\left(F^{\prime}\left(x_{j}\right)\right)\right\}=w\left(\underline{y}_{i} F^{\prime}\left(y_{i} / x_{j}\right)\right) \geqslant e_{s}^{P} .
$$

The property of being $s$-tautologous behaves in the same way by this rule. Namely, the algebra $P^{0}$ is obviously complete.

2. The other rules satisfy (Sem) because they preserve particular values. Namely, their interpretation always leads to identities in Post algebras. We quote them.

The rule of elimination of an existential quantifier $(\overline{\mathrm{Q}})$.

$$
\frac{\mathfrak{L}_{1}, \bar{y}_{i} F\left(y_{i}\right), \mathfrak{L}_{2}}{\mathfrak{L}_{1}, F^{\prime}\left(t_{j} / y_{i}\right), \mathfrak{L}_{2}, \bar{y}_{i} F^{\prime}\left(y_{i}\right)},
$$

where $t_{j}$ is the first member of sequence (4.9) such that the formula $F\left(t_{j}\right)$ does not yet appear in the construction of the diagram.

The elementary formulas of the form $A_{q}^{k}\left(t_{j_{1}} \ldots t_{j_{k}}\right)$, denoted by $A$, have to be decomposed according to the following rule (e):

$$
\frac{\mathfrak{L}_{1}, A, \mathfrak{L}_{2}}{\mathfrak{L}_{1}, K_{0}(A), \mathfrak{L}_{2} ; \mathfrak{L}_{1}, E_{1}, K_{1}(A), \mathfrak{L}_{2} ; \ldots ; \mathfrak{L}_{1}, E_{n-2}, K_{n-2}(A), \mathfrak{L}_{2}} .
$$

Rule (e) reflects the axiom of Post algebras (2.1). Indeed,

$$
\begin{array}{r}
w\left(\mathfrak{L}_{1}, A, \mathfrak{L}_{2}\right)=\inf \left\{w\left(\mathfrak{L}_{1}, K_{0}(A), \mathfrak{L}_{2}\right), w\left(\mathfrak{L}_{1}, \mathbb{E}_{1}, K_{1}(A), \mathfrak{L}_{2}\right), \ldots\right. \\
\left.\ldots, w\left(\mathfrak{L}_{1}, E_{n-2}, K_{n-2}(A), \mathfrak{L}_{2}\right)\right\} .
\end{array}
$$

The proof of the other rules is essentially the same. Namely, each rule is based upon appropriate identities (2.2)-(2.8) and many others very similar to those

$$
\begin{gathered}
\frac{\mathfrak{L}_{1}, J_{p}\left(E_{p}\right), \mathfrak{L}_{2}}{\mathfrak{L}_{1}, E_{n-1}, \mathfrak{L}_{2}} \quad\left(p \in I_{n}\right) \\
\frac{\mathfrak{L}_{1}, J_{p}\left(E_{q}\right), \mathfrak{L}_{2}}{\mathfrak{L}_{2}, E_{0}, \mathfrak{L}_{2}} \quad\left(p, q \in I_{n}, p \neq q\right)
\end{gathered}
$$$$
(\mathrm{JE})_{2}
$$ 
$(\mathrm{KE})_{1}$

$$
\frac{\mathfrak{L}_{1}, K_{p}\left(E_{p}\right), \mathfrak{L}_{2}}{\mathfrak{L}_{1}, E_{0}, \mathfrak{L}_{2}} \quad\left(p \in I_{n}\right) ;
$$

$(\mathrm{KE})_{2}$

$$
\frac{\mathfrak{L}_{1}, K_{p}\left(E_{q}\right), \mathfrak{L}_{2}}{\mathfrak{L}_{1}, E_{n-1}, \mathfrak{L}_{2}} \quad\left(p, q \in I_{n}, p \neq q\right) ;
$$

$$
\frac{\mathfrak{L}_{1}, J_{0}\left(J_{q}(F)\right), \mathfrak{L}_{2}}{\mathfrak{L}_{1}, K_{q}\left(F^{\prime}\right), \mathfrak{L}_{2}} \quad\left(q \in I_{n}\right) ;
$$

$$
\frac{\mathcal{L}_{1}, J_{p}\left(J_{q}\left(F^{\prime}\right)\right), \mathfrak{L}_{2}}{\mathfrak{L}_{1}, E_{0}, \mathfrak{L}_{2}} \quad\left(p, q \in I_{n}, p \neq 0, n-1\right)
$$

$(\mathrm{KK})_{1}$

$$
\frac{\mathfrak{L}_{1}, J_{n-1}\left(J_{q}(F)\right), \mathfrak{L}_{2}}{\mathfrak{L}_{1}, J_{q}(F), \mathfrak{L}_{2}} \quad\left(q \in I_{n}\right) ;
$$

$$
\frac{\mathfrak{L}_{1}, K_{0}\left(K_{q}\left(F^{\prime}\right)\right), \mathfrak{L}_{2}}{\mathfrak{L}_{1}, K_{q}\left(F^{\prime}\right), \mathfrak{L}_{2}} \quad\left(q \in I_{n}\right) ;
$$

$(\mathrm{KK})_{2}$

$(\mathrm{KK})_{3}$

$(\mathrm{JK})_{1}$

$(\mathrm{JK})_{2}$

$(\mathrm{JK})_{3}$

$(\mathbf{K J})_{1}$

$(\mathrm{KJ})_{2}$

$$
\frac{\mathcal{L}_{1}, K_{p}\left(K_{q}(F)\right), \mathfrak{L}_{2}}{\mathfrak{L}_{1}, E_{n-1}, \mathfrak{L}_{2}} \quad\left(p, q \in I_{n}, p \neq 0, n-1\right) ;
$$

$$
\frac{\mathfrak{L}_{1}, K_{n-1}\left(K_{q}\left(F^{\prime}\right)\right), \mathfrak{L}_{2}}{\mathfrak{L}_{1}, J_{q}\left(F^{\prime}\right), \mathfrak{L}_{2}} \quad\left(q \in I_{n}\right) ;
$$

$$
\frac{\mathfrak{L}_{1}, J_{0}\left(K_{q}(F)\right), \mathfrak{L}_{2}}{\mathfrak{L}_{1}, J_{q}\left(F^{\prime}\right), \mathfrak{L}_{2}} \quad\left(q \in I_{n}\right) ;
$$

$$
\frac{\mathfrak{L}_{1}, J_{p}\left(K_{q}(F)\right), \mathfrak{L}_{2}}{\mathfrak{L}_{1}, E_{0}, \mathfrak{L}_{2}} \quad\left(p, q \in I_{n}, p \neq 0, n-1\right)
$$

$$
\frac{\mathfrak{L}_{1}, J_{n-1}\left(K_{q}\left(F^{\prime}\right)\right), \mathfrak{L}_{2}}{\mathfrak{L}_{1}, K_{q}\left(F^{\prime}\right), \mathfrak{L}_{2}} \quad\left(q \in I_{n}\right) ;
$$$$
\frac{\mathfrak{L}_{1}, K_{0}\left(J_{q}\left(F^{\prime}\right)\right), \mathfrak{L}_{2}}{\mathfrak{L}_{1}, J_{q}\left(F^{\prime}\right), \mathfrak{L}_{2}} \quad\left(q \in I_{n}\right) ;
$$

$$
\frac{\mathfrak{L}_{1}, K_{p}\left(J_{q}(F)\right), \mathfrak{L}_{2}}{\mathfrak{L}_{1}, E_{n-1}, \mathfrak{L}_{2}} \quad\left(p, q \in I_{n}, p \neq 0, n-1\right) ;
$$

$(\mathbf{K J})_{3}$

(m)
$(\mathrm{Jj})_{2}$

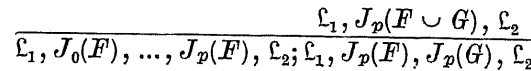
$\mathfrak{L}_{1}, J_{0}(F \cup G), \mathfrak{L}_{2}$ $\mathfrak{L}_{1}, J_{0}\left(\mathscr{F}^{\prime}\right), \mathfrak{L}_{2} ; \mathfrak{L}_{1}, J_{0}(G), \mathfrak{L}_{2} ;$

$\mathcal{L}_{2} ; \mathfrak{L}_{1}, J_{p}(F), J_{p}(G), \mathfrak{L}_{2} ; \mathfrak{L}_{1}, J_{0}(G), \ldots, J_{p}(G), \mathfrak{L}_{2}$

$$
\left(p \in I_{n}, p \neq 0, n-1\right) \text {; }
$$

$(\mathrm{Jm})_{1}$ $\frac{\mathfrak{L}_{1}, J_{n-1}(F \cup G), \mathfrak{L}_{2}}{\mathfrak{L}_{1}, J_{n-1}(F), J_{n-1}(G), \mathfrak{L}_{2}} ;$

$(\mathrm{Jm})_{2}$ $\frac{\mathfrak{L}_{1}, J_{0}(F \cap G), \mathfrak{L}_{2}}{\mathfrak{L}_{1}, J_{0}\left(F^{\prime}\right), J_{0}(G), \mathfrak{L}_{2}} ;$

$\frac{\mathfrak{L}_{1}, J_{p}(F \cap G), \mathfrak{L}_{2}}{\mathfrak{L}_{1}, J_{p}\left(F^{\prime}\right), \ldots, J_{n-1}\left(F^{\prime}\right), \mathfrak{L}_{2} ; \mathfrak{L}_{1}, J_{p}\left(F^{\prime}\right), J_{p}(G), \mathfrak{L}_{2} ; \mathfrak{L}_{1}, J_{p}(G), \ldots, J_{n-1}(G), \mathfrak{L}_{2}}$ $\left(p \in I_{n}, p \neq 0, n-1\right)$;

$(\mathrm{Jm})_{3}$

$(\mathbf{K})_{1}$ $\frac{\mathfrak{L}_{1}, J_{n-1}\left(F^{1} \cap G\right), \mathfrak{L}_{2}}{\mathfrak{L}_{1}, J_{n-1}\left(F^{\prime}\right), \mathfrak{L}_{2} ; \mathfrak{L}_{1}, J_{n-1}(G), \mathfrak{L}_{2}} ;$

$$
\frac{\mathfrak{L}_{1}, K_{0}(F \cup G), \mathfrak{L}_{2}}{\mathfrak{L}_{1}, K_{0}(\bar{F}), \bar{K}_{0}(G), \mathfrak{L}_{2}} ;
$$

$(\mathbf{K} \mathbf{j})_{2}$ $\mathfrak{L}_{1}, K_{p}(F \cup G), \mathfrak{L}_{2}$

$\frac{\mathfrak{L}_{1}, K_{0}(F), K_{p}(G), \mathfrak{L}_{2} ; \ldots ; \mathfrak{L}_{1}, K_{p}\left(F^{\prime}\right), K_{p}(G), \mathfrak{L}_{2} ; \ldots ; \mathfrak{L}_{1}, K_{p}(F), K_{0}(G), \mathfrak{L}_{2}}{\mathcal{L}}$ $\left(p \in I_{n}, p \neq 0, n-1\right)$;

$(\mathrm{Kj})_{3}$

$(\mathrm{Km})_{1}$

$$
\begin{gathered}
\frac{\mathfrak{L}_{1}, K_{n-1}(F \cup G), \mathfrak{L}_{2}}{\mathfrak{L}_{1}, K_{n-1}\left(F^{\prime}\right), \mathfrak{L}_{2} ; \mathfrak{L}_{1}, K_{n-1}(G), \mathfrak{L}_{2}} ; \\
\frac{\mathfrak{L}_{1}, K_{0}(F \cap G), \mathfrak{L}_{2}}{\mathfrak{L}_{1}, K_{0}\left(F^{\prime}\right), \mathfrak{L}_{2} ; \mathfrak{L}_{1}, K_{0}(G), \mathfrak{L}_{2}} ;
\end{gathered}
$$

$(\mathrm{Km})_{2}$ $\mathfrak{L}_{1}, K_{p}(F \cap G), \mathfrak{L}_{2}$

$\mathfrak{L}_{1}, K_{p}(\bar{F}), K_{n-1}(G), \mathfrak{L}_{2} ; \ldots ; \mathfrak{L}_{1}, K_{p}(F), K_{p}(G), \mathfrak{L}_{2} ; \ldots ; \mathfrak{L}_{1}, \bar{K}_{n-1}\left(F^{\prime}\right), K_{p}(G), \mathfrak{L}_{2}$

$$
\left(p \in I_{n}, p \neq 0, n-1\right) \text {; }
$$

$(\mathrm{Km})_{3}$

$$
\frac{\mathfrak{L}_{1}, K_{n-1}(F \cap G), \mathfrak{L}_{2}}{\mathfrak{L}_{1}, K_{n-1}\left(F^{\top}\right), \bar{K}_{n-1}(G), \mathfrak{L}_{2}} ;
$$

$$
\frac{\mathfrak{L}_{1}, J_{0}\left(\underline{y} \bar{F}^{\prime}\right), \mathfrak{L}_{2}}{\mathfrak{L}_{1}, \bar{y} J_{0}(\bar{F}), \mathfrak{L}_{2}}
$$

$\mathfrak{L}_{1}, J_{p}(y \vec{F}), \mathfrak{L}_{2}$

$\overline{\mathfrak{L}_{1}}, \bar{y} J_{p}\left(F^{\prime}\right), \mathfrak{L}_{2} ; \mathfrak{L}_{1}, \underline{y} K_{0}(\bar{F}), \mathfrak{L}_{2} ; \ldots ; \mathfrak{L}_{1}, \underline{y} K_{p-1}(\bar{F}), \mathfrak{L}_{2}$ 
$\frac{\mathfrak{L}_{1}, J_{n-1}(\underline{y} F), \mathfrak{L}_{2}}{\mathfrak{L}_{1}, y J_{n-1}(F), \mathfrak{L}_{2}}$

$(J \bar{Q})_{1}$ $\frac{\mathfrak{L}_{1}, J_{0}(\bar{y} F), \mathfrak{L}_{2}}{\mathfrak{L}_{1}, \underline{y} J_{0}\left(F^{\prime}\right), \mathfrak{L}_{2}}$

$(\mathrm{J} \overline{\mathrm{Q}})_{2}$$$
\frac{\mathfrak{L}_{1}, J_{p}(\bar{y} F), \mathfrak{L}_{2}}{\mathfrak{L}_{1}, \bar{y} J_{p}(F), \mathfrak{L}_{2} ; \mathfrak{L}_{1}, \underline{y} K_{p+1}(\bar{F}), \mathfrak{L}_{2} ; \ldots ; \mathfrak{L}_{1}, \underline{y} K_{n-1}(\bar{F}), \mathfrak{L}_{2}}
$$

$$
\left(p \in I_{n}, p \neq 0, n-1\right)
$$

$$
\frac{\mathfrak{L}_{1}, J_{n-1}\left(\bar{y} F^{\prime}\right), \mathfrak{L}_{2}}{\mathfrak{L}_{1}, \bar{y} J_{n-1}\left(\bar{F}^{\prime}\right), \mathfrak{L}_{2}}
$$

$$
\frac{\mathfrak{L}_{1}, K_{0}\left(\underline{y} F^{\prime}\right), \mathfrak{L}_{2}}{\mathfrak{L}_{1}, \underline{y} K_{0}(F), \mathfrak{L}_{2}}
$$

$$
\frac{\mathfrak{L}_{1}, K_{p}\left(\underline{y} F^{\prime}\right), \mathfrak{L}_{2}}{\mathfrak{L}_{1}, \underline{y} K_{p}(\bar{F}), \bar{y} J_{0}(\bar{F}), \ldots, \bar{y} J_{p-1}(F), \mathfrak{L}_{2}} \quad\left(p \in I_{n}, p \neq 0, n-1\right)
$$$$
\frac{\mathfrak{L}_{1}, K_{n-1}\left(y F^{\prime}\right), \mathfrak{L}_{2}}{\mathfrak{L}_{1}, \bar{y} K_{n-1}(\bar{F}), \mathfrak{L}_{2}}
$$

$$
\frac{\mathfrak{L}_{1}, K_{0}\left(\bar{y} F^{\prime}\right), \mathfrak{L}_{2}}{\mathfrak{L}_{1}, \bar{y} K_{0}\left(F^{\prime}\right), \mathfrak{L}_{2}}
$$

$(K \overline{\mathbf{Q}})_{2}$

$$
\begin{gathered}
\frac{\mathfrak{L}_{1}, K_{p}(\bar{y} F), \mathfrak{L}_{2}}{\mathfrak{L}_{1}, \underline{y} K_{p}(\bar{F}), \bar{y} J_{p+1}(\bar{F}), \ldots, \bar{y} J J_{n-1}(\bar{F}), \mathfrak{L}_{2}} \quad\left(p \in I_{n}, p \neq 0, n-1\right) ; \\
\frac{\mathfrak{L}_{1}, K_{n-1}(\bar{y} F), \mathfrak{L}_{2}}{\mathfrak{L}_{1}, \underline{y} \bar{K}_{n-1}\left(\overline{\left.F^{\prime}\right), \mathfrak{L}_{2}}\right.} .
\end{gathered}
$$

The definition of $D(F)$ is inductive. The list quoted above is both complete and minimal, i.e. there is exactly one rule according to which every formula of $\Lambda$ has to be decomposed if not indecomposable. Since the maximal number of possible premises is $2 n-3$, if $n \geqslant 3$, the elements of $\mathcal{D}\left(F^{\prime}\right)$ can be labeled by suitable sequences $\zeta_{i}=\left\langle z_{1}^{i}, \ldots, z_{k}^{i}\right\rangle$ composed of elements $z_{j}^{i} \in\{0, \ldots, 2 n-4\}$ as follows:

(i) $\mathfrak{L}_{\langle 0\rangle} \in \mathscr{D}(F)$ and it is the formula $F$ itself.

(ii) If $\mathfrak{\complement}_{\left\langle z_{1}, \ldots, z_{k}\right\rangle} \in \mathbb{D}(F)$, but it is an indecomposable or an $s$-fundamental sequence, then $\mathfrak{L}_{\left\langle z_{1}, \ldots, z_{k^{\prime}}, z_{k+1}\right\rangle}$ is not defined for any $z_{k+1}$. Such sequences are called end sequences of $\mathfrak{D}(\boldsymbol{F})$.

(iii) If $\mathfrak{L}_{\left\langle z_{1}, \ldots, z_{k}\right\rangle} \notin \mathfrak{D}\left(F^{\prime}\right)$, then $\mathfrak{L}_{\left\langle z_{1}, \ldots, z_{k}, z_{k+1}\right\rangle} \notin \mathfrak{D}\left(F^{\prime}\right)$ for any $z_{k+1}$.

(iv) If $\mathfrak{L}_{\left\langle x_{1}, \ldots, z_{k}\right\rangle} \in \mathfrak{D}\left(F^{\prime}\right)$, but it is neither indecomposable nor fundamental, then $\mathfrak{L}_{\left\langle z_{1}, \ldots, z_{k}\right\rangle}$ is a certain $\mathfrak{L}_{1}, G, \mathfrak{L}_{2}$ of $(4.8)$ while $\mathfrak{L}_{\left\langle z_{1}, \ldots, z_{k}, 0\right\rangle}$ will be the sequence $\mathfrak{L}_{1}, H_{1}^{1}, \ldots, H_{r_{1}}^{1}, \mathfrak{L}_{2}$ of $(4.8)$, i.e. the first premise of that rule, $\ldots, \mathfrak{L}_{\left\langle\varepsilon_{1}, \ldots, \varepsilon_{k}, q-1\right\rangle}$ will be the sequence $\mathfrak{L}_{1}, H_{1}^{q}, \ldots, H_{r_{q}}^{\alpha}, \mathfrak{L}_{2}$, i.e. the $q$ th premise and the sequences $\mathfrak{L}_{\left\langle z_{1}, \ldots, z_{k^{\prime}}, z_{k+1}\right\rangle}$ are not defined for any $z_{k+1} \geqslant q$.

$\mathscr{D}(F)$ is a tree if ordered with respect to the inclusion of initial segments with indexes $\zeta_{i}=\left\langle z_{1}^{i}, \ldots, z_{k}^{i}\right\rangle$ of its elements and, because of the rule $(\overline{\mathrm{Q}})$, not always a finite set.

5. Results. For every formula $F$ of $\Lambda$ the following four conditions are mutually equivalent:

(1) $F$ is $s$-valid,

(2) $F$ is s-tautologous,

(3) the diagram $D\left(F^{\prime}\right)$ is a finite set and each of its end sequences is $s$-fundamental,

(4) $F$ is provable in a modified Gentzen formalism.

The proof will be carried out according to the following diagram (6).

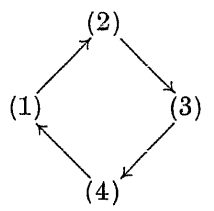

$(1) \rightarrow(2)$. Obvious.

$(2) \rightarrow(3)$. The proof is by contraposition.

Assume first that $\mathfrak{D}(F)$ is finite and that a certain end sequence of $D(F)$ is not $s$-fundamental. According to (Sem), $s$-validity ( $s$-tautologousness) of $F$ implies the $s$-validity ( $s$-tautologousness) of each $\mathfrak{L}_{\zeta} \in \mathbb{D}(F)$ and consequently of every end sequence. Now we prove that an end sequence $\mathfrak{C}_{\zeta}$ is not $s$-tautologous if it is not $s$-fundamental.

Such a sequence, being non-void and indecomposable, contains necessarily some formulas of the following three forms (5.1)-(5.3):

$$
\begin{gathered}
E_{r} \quad \text { with } \quad r<s, \\
K_{i_{1}}\left(A_{q}^{k}\left(t_{1}^{i_{1}} \ldots t_{k}^{i_{1}}\right)\right), \ldots, K_{i_{n}}\left(A_{q}^{k}\left(t_{1}^{i_{n}} \ldots t_{k}^{i_{n}}\right)\right),
\end{gathered}
$$

where $\left\langle t_{1}^{i_{p}}, \ldots, t_{k}^{i_{p}}\right\rangle \neq\left\langle t_{1}^{i_{q}}, \ldots, t_{k}^{i_{q}}\right\rangle$ if $p \neq q$ for $p, q=i_{1}, \ldots, i_{n}$,

$$
J_{m_{1}}\left(A_{q}^{k}\left(t_{1}^{i_{p}} \ldots t_{k}^{i_{p}}\right)\right), \ldots, J_{m_{r}}\left(A_{q}^{k}\left(t_{1}^{i_{p}} \ldots t_{k}^{i_{p}}\right)\right),
$$

where $m_{1}, \ldots, m_{r}$ do not exhaust the set $\{0, \ldots, n-1\}$ and where $i_{p}$ may appear among the $i_{1}, \ldots, i_{n}$ of (5.2), but then $i_{p} \neq m_{1}, \ldots, i_{p} \neq m_{r}$.

( ${ }^{\circ}$ Here " $\rightarrow$ " stands for "implies". 
In order to prove that such an $\mathfrak{L}_{\zeta}$ is not $s$-tautologous, we define an interpretation $w^{0}$ of $\Lambda$ within $\zeta$ and $P^{0}$ by taking the following canonical realization of terms:

$$
\begin{aligned}
w^{0}\left(c_{i}\right) & =c_{i}, \\
w^{0}\left(x_{i}\right) & =x_{i}, \\
w^{0}\left(\Phi_{q}^{k}\left(t_{i_{1}} \ldots t_{i_{k}}\right)\right) & =t_{j},
\end{aligned}
$$

where $t_{j}$ is that member of the sequence (4.9) which is of the form $\Phi_{q}^{k}\left(w^{0}\left(t_{i_{1}}\right) \ldots w^{0}\left(t_{i_{k}}\right)\right)$.

Since $w^{0}\left(E_{r}\right)=e_{r}^{0}$, let $w^{0}$ be defined for other elementary formulas as follows:

$$
a_{q}^{k}\left(w^{0}\left(t_{1}^{i_{r}}\right), \ldots, w^{0}\left(t_{k}^{i_{r}}\right)\right)=e_{i_{r}}^{0},
$$

where $i_{r}=i_{1}, \ldots, i_{n}$ if $A_{q}^{k}$ appears in $\mathfrak{L}_{\zeta}$ in the form (5.2). For a certain $A_{q}^{k}$ and the $i_{p}$ of (5.3) (if any such), $w^{0}$ has already been defined if $i_{p}$ occurs among the $i_{1}, \ldots, i_{n}$ of (5.2). If $i_{p}$ differs from each of them, let

$$
a_{q}^{k}\left(w^{0}\left(t_{1}^{i_{p}}\right), \ldots, w^{0}\left(t_{k}^{i_{p}}\right)\right)=e_{t}^{0},
$$

where $t$ is that member of the set $\{0, \ldots, n-1\}$ for which $t \neq m_{1}, \ldots, t \neq m_{r}$.

Outside $\mathfrak{L}_{t}$ let $w^{0}$ be defined in an arbitrary way. An elementary verification yields:

$$
\begin{aligned}
& w^{0}\left(K_{i_{r}}\left(A_{q}^{k}\left(t_{1}^{i_{r}} \ldots t_{k}^{i_{r}}\right)\right)\right)=k_{i_{r}}\left(e_{i_{r}}^{0}\right)=e_{0}^{0}, \\
& w^{0}\left(J_{m_{s}}\left(A_{q}^{k}\left(t_{1}^{i_{p}} \ldots t_{k}^{i_{p}}\right)\right)\right)=j_{m_{s}}\left(e_{t}^{0}\right)=e_{0}^{0} .
\end{aligned}
$$

Consequently, $w^{0}\left(\mathfrak{L}_{\zeta}\right)=e_{r}^{0}$ for a certain $r<s$.

Assume next that $\mathfrak{D}\left(F^{\prime}\right)$ is infinite. In this case $F^{\prime}$ is not $s$-tautologous either. Consider the set $\subseteq$ of all formulas of $D\left(F^{\prime}\right)$ which appear in at least one of those elements $\mathfrak{L}_{\zeta} \in \mathbb{D}(F)$ for which are defined the successors $\mathfrak{L}_{\eta}$ of every rank $\eta>\zeta$. $S$ is non-void because $\mathscr{D}(F)$ is infinite. We take again the canonical realization (5.4) and put

$$
\begin{gathered}
\boldsymbol{w}^{0}\left(\boldsymbol{A}_{q}^{k}\left(t_{j_{1}} \ldots t_{j_{k}}\right)\right)=\left\{\begin{array}{lll}
e_{i}^{0} & \text { if } K_{i}\left(A_{q}^{k}\left(t_{j_{1}} \ldots t_{j_{k}}\right)\right) \in \mathbb{G}, \\
e_{0}^{0} & \text { otherwise }
\end{array}\right. \\
\boldsymbol{w}^{0}\left(\boldsymbol{A}_{q}^{k}\left(t_{j_{1}} \ldots t_{j_{k}}\right)\right)= \begin{cases}e_{j}^{0}, j \neq i, & \text { if } J_{i}\left(\boldsymbol{A}_{q}^{k}\left(t_{j_{1}} \ldots t_{j_{k}}\right)\right) \in \mathbf{S}, \\
e_{0}^{0} & \text { otherwise }\end{cases}
\end{gathered}
$$

The supposition that $F$ is $s$-tautologous will lead to a contradiction. Namely, $w^{0}\left(F^{\prime}\right) \geqslant e_{s}^{0}$ also for the above interpretation, whence the set

$$
\mathscr{H}=\left\{G \in \Theta: w^{0}(G) \geqslant e_{8}^{0}\right\},
$$

a subset of $\mathcal{G}$, is non-void. But $\gamma\left(F^{\prime}\right)$ decreases through decomposition with respect to (Syn). On account of (Sem) we may expect further elements in $\mathfrak{H}$, i.e. there is a certain formula $H \in \mathcal{H}$ of minimal order. If this $H$ is indecomposable and of the form

$$
K_{i}\left(A_{q}^{k}\left(t_{j_{1}} \ldots t_{j_{k}}\right)\right) \quad \text { or } \quad J_{i}\left(A_{q}^{k}\left(t_{j_{1}} \ldots t_{j_{k}}\right)\right)
$$

then $w\left({ }^{0} H\right)=e_{0}^{0}$ by definitions (5.5), resp. (5.6). On the other hand, $w^{0}(H) \geqslant e_{s}^{0}$ because $H \in \mathcal{H}$. This is the contradiction.

It remains to prove that the set $R$ of all indecomposable formulas of $H$ contains some formulas of the form (5.7) if $F$ is supposed to be $s$-tautologous.

Indeed, $E_{i} \notin \mathcal{R}$. Otherwise $i<s$ would imply $w^{0}\left(E_{i}\right)<e_{s}^{0}$, whence $E_{i} \notin \mathcal{J}$ and, consequently, $E_{i} \notin \mathcal{R}$. If $i \geqslant s$, then $E_{i} \in \mathfrak{L}_{\zeta}$ for a certain $\zeta$. Therefore $\mathcal{L}_{t}$ would be an $s$-fundamental sequence of $\mathcal{D}\left(F^{\prime}\right)$, whence an end sequence of it. But no formula from any end sequence of $\mathfrak{D}\left(F^{\prime}\right)$ belongs even to $\mathrm{G}$.

The other properties of the set $\mathcal{R}$ are:

(i) if a certain $G \in \mathcal{R}$ belongs to $\mathcal{L}_{\xi}$, so does the same $G$ for each $\mathcal{L}_{\xi}$ with $\xi>\zeta$ (by the definition of the diagram and the fact that $R \subset g$ );

(ii) if a certain $K_{i}\left(A_{q}^{k}\left(t_{j_{1}} \ldots t_{j_{k}}\right)\right) \in \mathcal{R}$, then for any $j \neq i K_{j}\left(A_{q}^{k}\left(t_{j_{1}} \ldots t_{j_{k}}\right)\right)$ $\notin R$. In the opposite case both formulas would appear together in a certain $\mathfrak{L}_{\eta}$, which is a common successor of $\mathfrak{L}_{t}$ and $\mathfrak{L}_{\xi}$ in $D(F)$, whence $\mathfrak{L}_{\eta}$ would be an $s$-fundamental sequence and therefore an end sequence of $\mathfrak{D}\left(F^{\prime}\right)$. This, however, is impossible.

(iii) if a certain $K_{i}\left(A_{q}^{k}\left(t_{j_{1}} \ldots t_{j_{k}}\right)\right) \in \Re$ together with some $J_{m_{1}}\left(A_{q}^{k}\left(t_{j_{1}} \ldots t_{j_{k}}\right)\right)$, $\ldots, J_{m_{r}}\left(A_{q}^{k}\left(t_{j_{1}} \ldots t_{j_{k}}\right)\right)$, then neither $i=m_{1}, \ldots, i=m_{r}$ nor $m_{1}, \ldots, m_{r}$ exhaust the set $\{0, \ldots, n-1\}$. In other words, such a $j \neq i$ as given in (5.6) can always be chosen.

The assumption that $H$ is decomposable also leads to a contradiction. If a certain rule of the form (4.8) has to be applied to such an $H$, then, as we know by (4.7) and (Sem), for every $i, 1 \leqslant i \leqslant q$, there is a $j, 1 \leqslant j$ $\leqslant r_{i}$, such that $G_{j}^{i} \in \mathcal{H}$ if $H \in \mathcal{H}$. However, $\gamma\left(G_{j}^{i}\right) \leqslant \gamma(H)$ for every $i$ and $j$ because of (Syn). It turns out that $H$ is not of minimal order.

Finally let $H$ be of the form $\bar{y}_{i} G\left(y_{i}\right)$. Let $H$ belong to $\complement_{\zeta}$ with minimal $\zeta$, .e. the rule $(\overline{\mathbf{Q}})$ has been applied to $H$ for the first time and with $t_{j}$ as term. By the supposition $\bar{y}_{i} G\left(y_{i}\right) \in \mathcal{K}$, i.e.

$$
e_{s}^{0} \leqslant w^{0}\left(\bar{y}_{i} G\left(y_{i}\right)\right)=\sup _{w^{\prime} \in W\left(w^{0, j}\right)}\left\{w^{\prime}\left(G\left(x_{j} / y_{i}\right)\right)\right\} .
$$

Hence there is a successor $\mathfrak{L}_{\xi}$ of $\mathcal{L}_{\zeta}$ in $\mathfrak{D}\left(F^{\prime}\right)$ (not necessarily the immediately first one) of the form

$$
\mathfrak{L}_{1}^{\prime}, G\left(t_{k} \mid y_{i}\right), \mathfrak{L}_{2}^{\prime}, \bar{y}_{i} G\left(y_{i}\right),
$$


but such that $w^{0}\left(G\left(t_{k_{k}} / y_{i}\right)\right) \geqslant e_{s}^{0}$. Namely, $P^{0}$ is totally ordered. Therefore $G\left(t_{k} \mid y_{i}\right) \in$ He. But $\gamma\left(G\left(t_{k} / y_{i}\right)\right)<\gamma\left(\bar{y}_{i} G\left(y_{i}\right)\right)$. This violates the minimality of $\gamma(H)$.

$(3) \rightarrow(4)$. We describe first what kind of Gentzen formalism will be used. Every $s$-fundamental sequence is thought of as a provable sequence $\left({ }^{7}\right)$. We assume that to every rule of decomposition (of elimination of a connective or a quantifier) of the form

$$
\frac{\mathfrak{L}}{\mathfrak{L}_{1} ; \ldots ; \mathfrak{L}_{q}}, \quad q \geqslant 1 \text {, }
$$

corresponds a rule of inference (of the introduction of that connective or quantifier) of the form

$$
\frac{\mathfrak{L}_{1}, \ldots, \mathfrak{L}_{q}}{\mathfrak{L}} .
$$

The proof of a formula $F$ is a finite sequence of sequences of formulas with the property that its last member is the formula $F$ itself and for each member either of the following two conditions holds: (i) it is an $s$-fundamental sequence, (ii) it is obtained from some preceding sequences through the application of a rule of inference (5.8).

A formula is said to be provable if there is a proof of it.

Now to the proof itself. Rearrange the elements of $\mathfrak{D}(F)$ in a sequence

$$
\mathfrak{L}_{\zeta_{1}}, \ldots, \mathfrak{L}_{b_{k}}, \ldots, \mathfrak{f}_{t_{n}}
$$

with respect to the decreasing lengths of their indexes $\zeta_{i}$ and those of equal lengths lexicographically. The sequence (5.9), when supplied with a suitable commentary, is the proof of the formula $F$. The proof of this fact is essentially the same as that used in [3]. Namely, (5.9) is a finite sequence of sequences by supposition and $\mathcal{L}_{\zeta_{n}}$ is the formula $F$ itself. The sequence $\mathcal{L}_{\zeta_{1}}$ is $\delta$-fundamental because $\zeta_{1}$ is of maximal length, i.e. $\mathfrak{L}_{\hbar_{1}}$ is an end sequence of $\mathfrak{D}(F)$. For every $k, 1<k \leqslant n$, and the corresponding $\zeta_{k}$ of length, say, $p$ the following statement holds: either $\zeta_{k}$ is not a proper initial segment of any of the sequences of length $p+1$ which are given in (5.9) by $\zeta_{j_{1}}, \ldots, \zeta_{j_{s}}$ and where $\mathfrak{L}_{\xi_{j_{1}}}, \ldots, \mathfrak{L}_{\xi_{j_{s}}}$ precede $\mathfrak{L}_{\zeta_{k}}$ in (5.9), or $\zeta_{k}$ is a proper initial segment of $q, 1 \leqslant q \leqslant s$, such sequences. In the first case none of the sequences $\mathfrak{L}_{b_{j_{i}}}$, where

$$
\zeta_{j_{i}}=\left\langle z_{1}^{j_{i}}, \ldots, z_{p}^{j_{i}}, z_{p+1}^{j_{i}}\right\rangle \quad \text { for } \quad i=1, \ldots, s,
$$

(7) E.g. $E_{i}$, for $i \geqslant s$, is a provable sequence that is also a provable formula. If $\mathfrak{L}_{1}, A, \mathfrak{L}_{2}$ is an $s$-fundamental sequence, so is $\mathcal{L}_{1}, A, B, \mathfrak{L}_{2}$. The same is true of $\mathfrak{L}_{1}, A, B, \mathcal{L}_{2}$ and $\mathfrak{L}_{1}, B, A, \mathfrak{L}_{2}$. Therefore we neglect the structural rules of inference. is defined for any $z_{p+1}^{j_{j}}$, i.e. $\mathcal{L}_{t_{k}}$ is an end sequence of $D(F)$ and, consequently, $s$-fundamental. In the second case we have

$$
\begin{aligned}
\zeta_{k} & =\left\langle z_{1}^{k}, \ldots, z_{p}^{k}\right\rangle, \\
\zeta_{j_{i}} & =\left\langle z_{1}^{k}, \ldots, z_{p}^{k}, z_{p+1}^{j_{i}}\right\rangle \quad \text { for } \quad i=1, \ldots, q .
\end{aligned}
$$

Hence $\mathfrak{L}_{\zeta_{k}}$ is the conclusion of that rule of inference which corresponds to the rule of decomposition just here applied.

$(4) \rightarrow(1)$. The $s$-fundamental sequences are obviously $s$-valid (cf. (2.2), $(2.3),(2.4)$ and (4.7)). With respect to (4.7), (4.8), (Sem) and (5.8) $s$-validity is preserved under the rules of inference. Hence every provable formula is $s$-valid.

6. Implication. No implication has been used so far. However, if we put

$$
x \rightarrow y=\bigwedge_{i>j} k_{i}(x) \vee k_{j}(y),
$$

we are able to interpret each formula of the form $A \Rightarrow B$ within every Post algebra of order $n$ taking

$$
w(A \Rightarrow B)=w(A) \rightarrow w(B)\left({ }^{8}\right) .
$$

The decomposition of formulas of the form $A \Rightarrow B, K_{i}(A \Rightarrow B)$, and $J_{i}(A \Rightarrow B)$ becomes a matter of simple computation because there is a perfect normal form for each of the functions $x \rightarrow y, k_{i}(x \rightarrow y)$ and $j_{i}(x \rightarrow y)$.

There is an axiomatic stipulation of propositional calculus built up so as to ensure that the Lindenbaum-Tarski's algebra of formulas is a countable Post algebra of order $n$, which justifies the above implication. The usual detachment rule is sufficient to develop such a stipulation. The completeness theorem follows from the fact that the quotient algebra modulo a prime ideal of order zero is an $n$-element Post algebra. These results, however, lie outside the scope of the present article and they deserve particular treatment.

\section{References}

[1] G. Epstein, The lattice theory of Post algebras, Trans. Amer. Math. Soc. 95 (1960), pp. 300-317.

[2] G. Gentzen, Untersuchungen über das logische Schliessen I, Math. Zeitschr. 39 (1934), pp. 176-210; II, ibidem, pp. 250-268.

${ }^{(8)}$ For $n=2$ the above definition becomes just $\neg x \vee y$. This operator possesses many pleasant properties, e.g. $x \leqslant y$ if and only if $x \rightarrow y=e_{n-1}$. If $x \rightarrow y \geqslant e_{s}, s \neq 0$, $n-1$, then $x \rightarrow y=e_{n-1}$ because the element $x \rightarrow y$ is not comparable with $e_{1}, \ldots, e_{n-2}$. 
[3] V. G. Kirin, Gentzen's method for the many-valued propositional calculi, Zeitschr. Math. Logik Grundlagen Math. 12 (1966), pp. 317-332.

[4] - Ujecia algebraiczne $i$ Gentzenowskie logik Posta, (Dysertacja), Warszawa 1966, pp. IV +114.

[5] A. Mostowski, Axiomatizability of some many valued predicate calculi, Fund. Math. 50 (1961), pp. 165-190.

[6] H. Rasiowa and R. Sikorski, On the Gentzen Theorem, Fund. Math. 48 (1959), pp. 57-69.

[7] T. Traczyk, Axioms and some properties of Post algebras, Colloq. Math. 10 (2) (1963), pp. 193-209.

Reçu par la Rédaction le 2. 6.1967

\section{On ordered topological spaces}

by

R. Du d a (Wrocław)

1. A topological space $X$ is called ordered if there exists a transitive relation $\zeta$, called order in $X$, satisfying the following two conditions (see [2], p. 38):

(i) If $x, y \in X$, then one and only one of the relations $x<y, x=y$, $y<x$ holds.

(ii) If $x, y \in X$ and $x>y$, then there exist neighbourhoods $U(x)$ of $x$ and $U(y)$ of $y$ such that $x<y^{\prime}$ and $x^{\prime} \zeta y$ whenever $x^{\prime} \epsilon U(x)$ and $y^{\prime} \in U(y)$.

Condition (ii) can be, as is easy to observe, replaced by the following one

(ii') If $p \in X$, then the sets $\{x \in X: x<p\}$ and $\{x \in X: p \nmid x\}$ are both open in $X$.

In what follows an ordered space will always mean an ordered topological space.

There are many examples of ordered spaces. Such are, for instance, the diagrams $\{(x, f(x)): x \in R\}$, where $f: R \rightarrow Y$ is any function mapping the real line $R$ into a topological space $Y$, with the topology inherited from $R \times Y$. A great variety of such diagrams, interesting from the topological point of view, can be found already in the case of $Y=R$, cf. [14]. Another set of examples of ordered spaces is provided by metric separable spaces whose all quasicomponents are single points (cf. [10], II, p. 93; see also the Remark following Theorem 6 of this paper).

Ordered spaces have several interesting properties (for instance, they are all Hausdorff spaces) and they have already been studied to some extent, e.g. in [2]. The aim of the present paper is to conduct this study further.

Thus, in part 2 of this paper we shall show that in ordered spaces quasicomponents coincide with components (Theorem 1) and we shall discover a close affinity between ordered connected spaces on one hand and irreducibly connected spaces on the other hand (Theorem 3), both results to be applied later. 